\title{
Step-wise Approach to Investigate the Impact of Energy Transition on Voltage Dips in the Dutch Electricity Grid
}

\author{
R. Torkzadeh ${ }^{1}$, R. L. E. Peters ${ }^{1}$, V. Ćuk ${ }^{1}$, J.B.M. van Waes ${ }^{2}$ and J. F. G. Cobben ${ }^{1}$ \\ ${ }^{1}$ Department of Electrical Engineering, Eindhoven Technical University (TU/e), \\ $5600 \mathrm{MB}$, Eindhoven, The Netherlands. \\ Phone number: +31 (0)40 247 6667, e-mail: \{r.torkzadeh, v.cuk and_.f.g.cobben\}@tue.nl and \\ r.l.e.peters@student.tue.nl \\ 2 TenneT TSO B.V. Utrechtseweg 310, Arnhem, The Netherlands. \\ e-mail: Jeroen.van.waes@tennet.eu
}

\begin{abstract}
The energy transition and therewith generation shift from conventional centralized synchronous generation to dispersed renewable energy sources drives huge changes in the emerging power grids. This paper proposes a step-wise approach for analysing the impacts of energy transition on voltage dips in the future grid, including the challenges. Based on three consecutive years of measurement data, an analysis was made on the type of events that have occurred in the Dutch HV and EHV grids. Finally, several events have been selected that will be used for model validation.
\end{abstract}

Key words. Power quality, energy transition, voltage dips, power quality measurements.

\section{Introduction}

In order to minimize the impacts of climate change and to achieve the environmental protection goals, significant non-synchronous renewable energy sources (RESs) will be integrated into the grid. This integration is a part of the socalled energy transition (ET) that is happening almost all around the world [1], including the Netherlands.

The Dutch grid-code defines the assessment criteria with respect to power quality. Voltage dips are an important topic due to their huge impact on network operators and connected-parties in both transmission and distribution grids [2].

The generation shift from conventional centralized synchronous generation to non-synchronous RESs would impact the frequency response of the power system, as well as, the short-circuit levels of the system. The former has been considered in many research and regulations [3][5], to minimize the impacts on the inertial response of the system. However, there are limited number of works focusing on the latter. The changes in the short-circuit level of the system due to reduction in the share of synchronous generation in the total generation would influence the propagation of voltage dips through the grid, which may affect more connected-parties.

Voltage dips can be characterized by their duration, retained voltage, number of affected phases and their occurrence frequency. Table I shows the classification of voltage dips regarding their retained voltage and duration according [2].

The impacts of an increase in the amounts of wind power generation and distributed energy resources (DERs) mainly connect to distribution networks on the voltage dip frequency of occurrence are assessed in [6] and [7], respectively. According to these researches, the frequency of voltage dips will increase in both cases. However, the other aspects of voltage dips have not been considered in these researches.

The authors of [8] have assessed the effects of reduced rotating inertia on the voltage dip propagation in the transmission system of Germany, for various futuristic cases. According to [8] the voltage dip propagation would alter due to the changes in the short-circuit level after closure of nuclear power plants. An event during a generation dispatch with high RES penetration could affect more connected-parties, in comparison to the same event, with more conventional synchronous generations in service.

The study assumes that the network topology will not change in futuristic scenarios, and the scenarios were built only by up-scaling the load and generation. However, to understand the impacts of the ET on voltage dips, it is essential to define the futuristic scenarios, which include grid reinforcements.

This paper proposes an approach for conducting this impact analysis. Moreover, the registered events by power quality meters (PQMs) in the Dutch EHV, HV and MV grids for the past three years are analysed as a part of this research. The aims of this paper are:

- Propose a step-wise approach for assessing the effects of the ET on voltage dips, by considering the challenges, bottlenecks and possible solutions.

- Illustrate the current status of Dutch electricity network from the voltage dip perspective via analysing logged events by PQMs.

The rest of the paper is organized as follows:

Section 2 discusses the proposed approach for analyzing the impacts of the ET on the voltage dips. Section 3 summarizes the analysis of recorded voltage dips over a 3 -year period and Section 4 concludes with a summary. 
Table I. The voltage dip categories according to [2].

\begin{tabular}{|c|c|c|c|c|}
\hline \multirow{2}{*}{$\begin{array}{c}\text { RETAINED } \\
\text { VOLTAGE }(\%)\end{array}$} & \multicolumn{4}{|c|}{ DURATION $(m s)$} \\
\hline & $\begin{array}{c}10 \text { to } \\
200\end{array}$ & $\begin{array}{c}200 \text { to } \\
500\end{array}$ & $\begin{array}{c}500 \text { to } \\
1000 \\
\end{array}$ & $\begin{array}{c}1000 \text { to } \\
5000\end{array}$ \\
\hline $80 \% \leq \mathrm{u}<90 \%$ & & \multirow{2}{*}{ Class A } & & \\
\hline $70 \% \leq \mathrm{u}<80 \%$ & & & \multirow{4}{*}{\multicolumn{2}{|c|}{ Class C }} \\
\hline $40 \% \leq \mathrm{u}<70 \%$ & & \multirow{3}{*}{ Class B2 } & & \\
\hline $5 \% \leq \mathrm{u}<40 \%$ & \multirow{2}{*}{ Class B1 } & & & \\
\hline $\mathrm{u}<5 \%$ & & & & \\
\hline
\end{tabular}

\section{The Proposed Approach}

To investigate the impacts of the ET on the power quality of emerging power grids, it is important to consider various aspects. The initial focus will be on voltage dips. The development of models for the current and future grid, identification of methods for studying the voltage dip phenomena, and determination of the simulation platform, to name a few. Thus, it is essential for the proposed stepwise approach to cover all these aspects.

Figure 1 shows a flowchart diagram of the proposed approach. This scheme contains four major orientations, namely, "existing grid", "future grid", "events and contingencies", and "comparison", which are illustrated by blue, green, yellow and purple areas, respectively. Each of these orientations are explained in detail in the followings.

\section{A. Analysis of Existing Grid Model}

The aim of this orientation is to identify the parameters of influence and prepare and validate a model for the existing grid. This orientation focuses on defining a Base Case as the representative for the current status of the Dutch grid. The simulations result of the Base Case will be compared to the ones from the grid after the ET, in order to illustrate the changes.

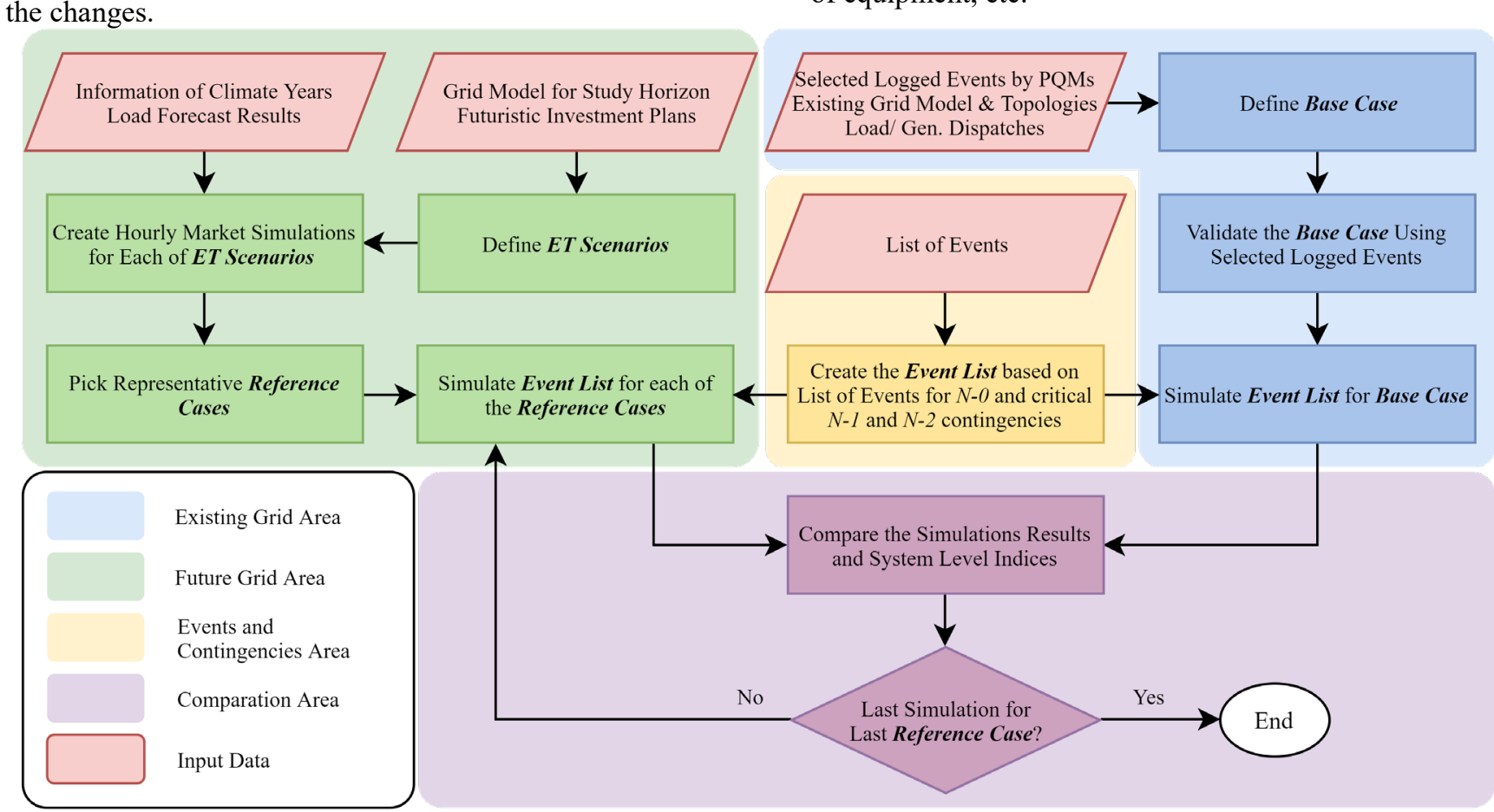

As part of this orientation, the logged data by PQMs will be used to verify the model. A selection of events that occurred in the grid will be simulated using the snapshots of the system (pre-, post- and during the event). The chosen events are the ones that have caused the most severe voltages dips, along with, been registered by as many as PQMs in the (E)HV/MV grids. The comparison of the simulation results versus the measurements from PQMs identifies the level of trust to the model. The event selection for model validation process is discussed in more details in Section 3.

\section{B. Definition of Future Grid}

The aim of this orientation is to define the ET, in more detail. Due to the uncertainties of planning, it is crucial to consider a spectrum of scenarios. Additionally, it is important to consider multiple horizons to assess the midterm and long-term evolution of the grid.

Thus, in this research several ET scenarios are defined for the mid-term horizon of 2025 and long-term horizon of 2030. These ET scenarios are defined based on available energy investment plans (IP) for the 2020, 2025 and 2030 horizons in The Netherlands by TenneT, the Dutch transmission system operator [9].

In [9], the most-probable scenarios for ET in The Netherlands are explained for both electricity and gas networks from a high-level perspective. According to the possibilities mentioned in IP, some critical aspects of the ET are defined, e.g. the installed capacity of each generation type, estimated number of electric vehicles, number of consumers, installed capacity of energy storages, etc. These ET scenarios include both generation-shift and grid reinforcements. The grid reinforcement mainly refers to establishment of new (E)HV substations and transmission lines, rating upgrade of equipment, etc.

Fig. 1. The flowchart diagram of the proposed approach to study the impacts of the ET on voltage dip propagation. 
According to the IP, there are three possible evolutions for Dutch energy (electricity and gas) networks which are:

- Climate Agreement (CA): The most probable evolution.

- Fundamentals for System Integration (FSI): The evolution with most installed capacity of renewables.

- Alternative Transition (AT): A moderated evolution between CA and FSI.

Figure 2 depicts an overview of these possible ET Scenarios for 2020, 2025 and 2030 according to the IP.

For each of these energy transition scenarios, there is an enormous variety of grid states (i.e. various load conditions, generation dispatches, renewable in-feeds, network configuration, etc.). In addition, the number of cases increase drastically if the N-1 and N-2 conditions are going to be considered. Thus, the computational burden for simulating various events becomes the main challenge of this orientation.

A practical solution to ameliorate the scenario creation process is to consider a limited number of most representative cases, instead of all possible scenarios. These representative Reference Cases are used as the future grid models for the simulations.

Figure 3 illustrates the proposed Reference Cases creation method. As it is depicted, for each of the ET Scenarios, i.e. "CA-2020", "CA-2025", "CA-2030", "FSI-2025", "FSI2030" and "AT-2030", the winter peak load and the summer valley load are applied for the loading condition, as the highest and lowest values of the system total load, respectively. Each of the ET Scenarios describes the physical interconnection of the system and includes grid configuration, installed capacity of generation and load, etc.

It is assumed that the occurrence of dips is independent of the loading or a dispatch scenario. To cover the most common state of the gird, the most frequent loading condition and dispatch scenario are needed to be included, as this is the condition under which events are most likely to occur. An addition two extreme renewable penetration levels could be considered to have the maximum fluctuations in the short-circuit level. The winter peak and summer valley loads are the most extreme cases and not necessarily the most common loading conditions of the network.

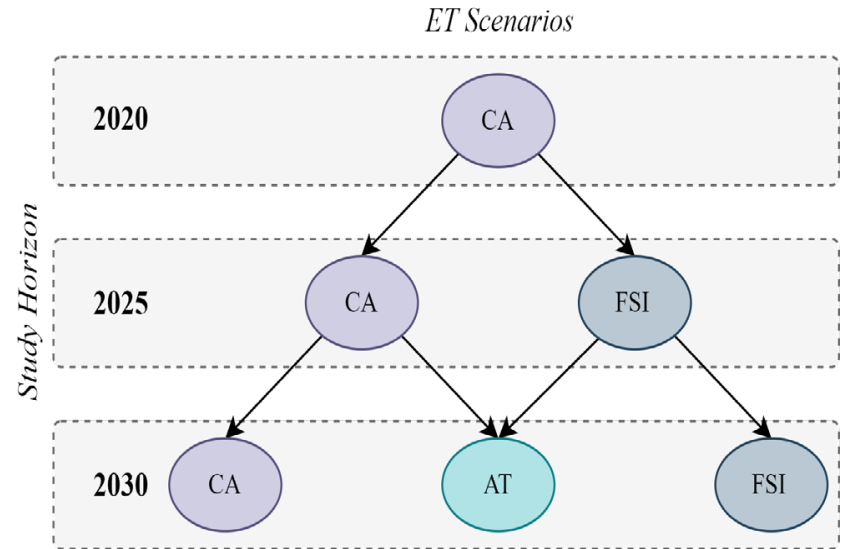

Fig. 2 Possible Dutch energy grid evolutions after ET.

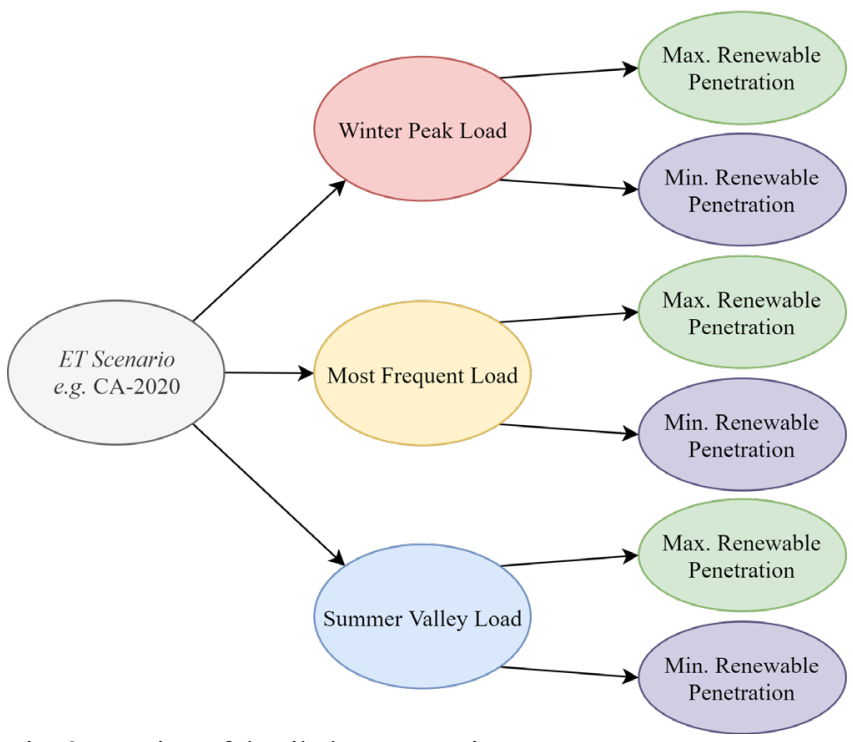

Fig. 3 Creation of detailed ET scenarios.

To sum up the future grid orientation, the outcome of this orientation will be various Reference Cases that are the most representative states of the future grid and will be used for the simulations to illustrate the impacts of the ET.

\section{Definition of Areas of Interest and Credible Events}

The aim of this orientation is to identify the areas of the grid with the greatest changes. This includes a preparation of an Event List which is used to compare the grid behaviour before and after the ET. The short circuit power affects the voltage dip propagations through the network.

For investigating the former goal, variations in threephase short-circuit level between a futuristic case in 2030 and the grid in 2018 are determined. It is important to distinguish maximum and actual fault current levels.

In general, the maximum short-circuit level shows an increase in the EHV grid and most of areas in the HV grid. This is due to grid reinforcements to facilitate the transmission of renewable energy and to facilitate the international energy markets.

The method for calculating the maximum short-circuit level, considers all the generation units in-service and participating in the fault current. For some areas in the $\mathrm{HV}$ grid there will be a decrease in the maximum shortcircuit level due to a closure of centralized power plants. The actual short-circuit levels of the system are likely to fluctuate due to the variations in the mix of generation. To achieve the latter goal of this orientation the list of most probable/critical events with their characteristics should be prepared to form the Events List. The events within this list will be simulated for both existing and futuristic grids.

To conclude this orientation, the outcomes will be a set of buses that are within the areas of the grid with the most changes; and an Events List to be considered in the simulations.

D. Comparison of Reference Cases with "2020" Base Case 
The simulation results for all the events within the Event List on each of Reference Cases are compared with the corresponding simulations results for the Base Case.

Several system level indices such as Sag Propagation Index (SPI) and Sag Energy Index (SEI) could be used to illustrate and quantify the impacts of the ET on the voltage dips. For instance, the propagation area of the voltage dip or the number of affected connected-parties for a specific event can be compared before and after the ET.

\section{E. Assumptions}

To define the boundaries of this research, several assumptions should be made. Firstly, the time-horizon of the study for each grid (EHV/HV/MV); location and number of connected parties, generation plants and RESs; and details of adjacent networks will be considered aligned with capacity planning calculations process in TenneT.

Secondly, the total number faults/events per year per $\mathrm{km}$ for the grid after ET is assumed to be equal to the existing grid, and protection devices are considered to work properly based on defined settings and configuration, with no mal-operation. Thirdly, the fault ride through (FRT) capability should be considered according [5].

\section{Analysis of Events Logged by PQMs}

In the Netherlands every connected (E)HV connectedparty (excluding the distribution network operators) have a PQM. Over 125 PQMs are widely installed at different buses of the system. Moreover, in MV grids, a huge number of meters are installed. Amongst others voltage dips are registered by these PQMs.

These measurements are used to obtain an insight into the current status of the system from voltage dip perspective and to select an event to be used for model validation.

Figure 5 shows all registered voltage dip events for three consecutive years (from 01-07-2016 to 01-07-2019. Each event is illustrated with one red dot, which demonstrates the logged retained voltage of the PQM with the lowest value among all the PQMs that has registered the events versus the duration of corresponding event.

Approximately $75 \%$ of all dip-events were caused by a 1 phase-to-earth fault. Most important are the severe voltage dips (Class B1, B2 and C according to [2]) of which approx. 5\% (25 events) were registered during these 3 years.

An analysis of these severe dips reveals that approx. 4\% of severe dips originate from single-phase faults $68 \%$ by twophase faults $20 \%$ by three-phase faults and $8 \%$ have unclear origins. The results of this analysis are depicted in Fig. 6. This analysis is the first step toward identification of the candidate event for model verification.

However, the events originated from two-phase faults are the most frequent ones, but due to huge horizontal (same voltage level) and vertical propagation of voltage dips originated by three-phase faults, the candidate should be chosen from them.

Table II indicates a short-list for the events that were originated by three-phase faults. On one hand, Event 3 and Event 5 are the most severe dips within the list due to values of their retained voltage and duration (B2 category); and from the other hand; only three of these events have the wide propagation in both (E)HV and MV networks (Events 2, 3 and 4), which can be understood by comparing the number of PQMs that has registered the event on each grid.

However, the number of PQMs that have registered an event is insufficient to prove the wide propagation of a voltage dip event due to the fact that PQMs may not be dispersed through the grid homogenously.

In order to overcome this challenge, the spatial propagation of minimum retained voltage by all PQMs that have recorded the events for Event 2, 3 and 4 in HV and MV grids are shown in Fig 7(a) to 7(f), respectively. This is clear that both Event 3 and Event 4 have a wide propagation in both HV and MV networks. Therefore, by considering the both criteria (i.e. severity of the event and number of PQMs that have registered the events in (E)HV and MV grids), Event 3 is selected as the candidate for model verification.

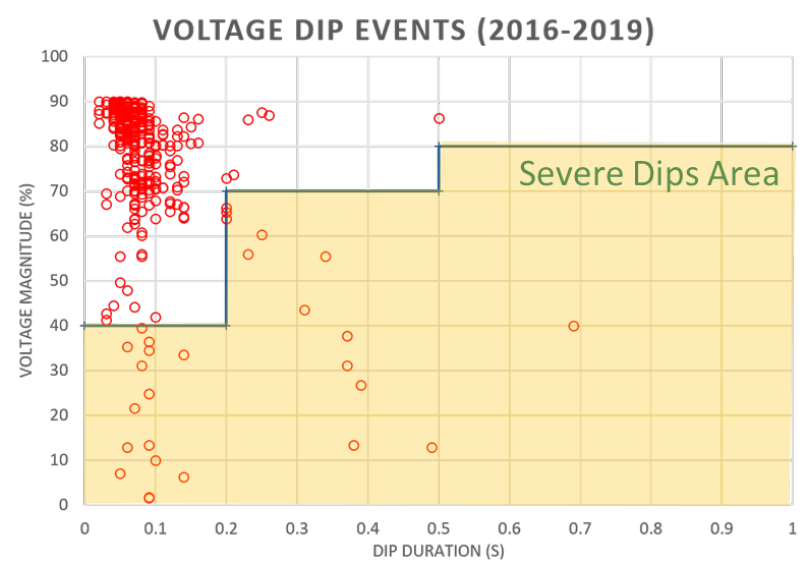

Fig. 4 Logged voltage dip events from 01-07-2016 to 01-072019.

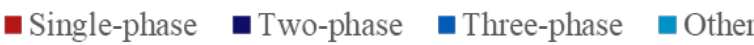

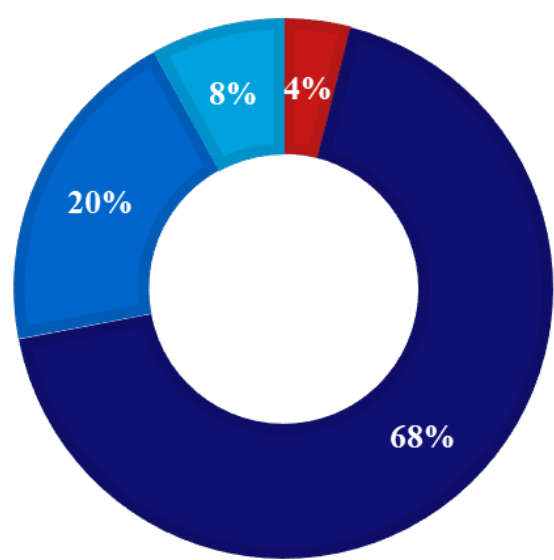

Fig. 5. An overview on the origins of severe voltage dip events.

Table II. The short list of candidate events for model verification

\begin{tabular}{|c|c|c|c|}
\hline $\begin{array}{c}\text { Event } \\
\text { No. }\end{array}$ & $\begin{array}{c}\text { Retained } \\
\text { Voltage (\%) }\end{array}$ & $\begin{array}{c}\text { No. of PQM } \\
\text { Registers at (E)HV }\end{array}$ & $\begin{array}{c}\text { No. of PQM } \\
\text { Registers at MV }\end{array}$ \\
\hline 1 & 33.52 & 3 & 0 \\
\hline 2 & 13.35 & 18 & 27 \\
\hline 3 & 31.08 & 11 & 37 \\
\hline 4 & 60.36 & 11 & 30 \\
\hline 5 & 26.78 & 18 & 3 \\
\hline
\end{tabular}




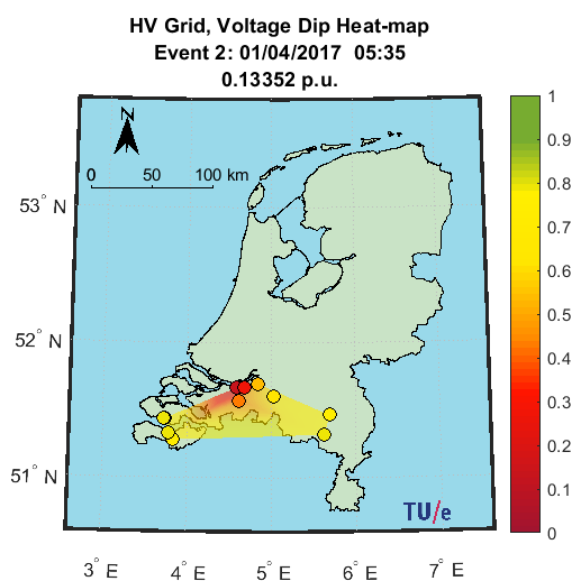

(a)

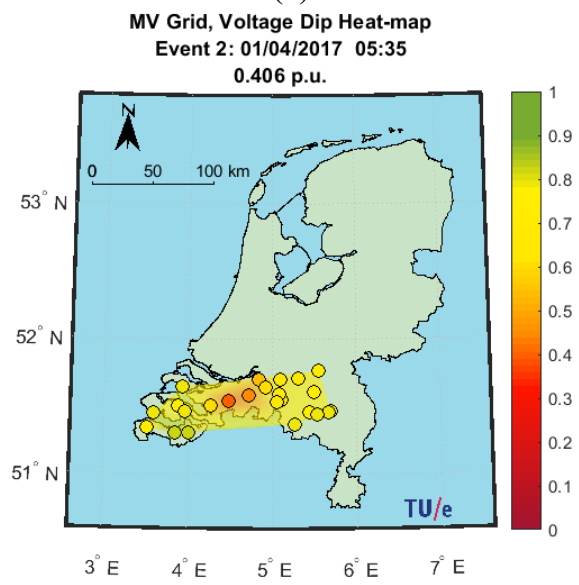

(b)

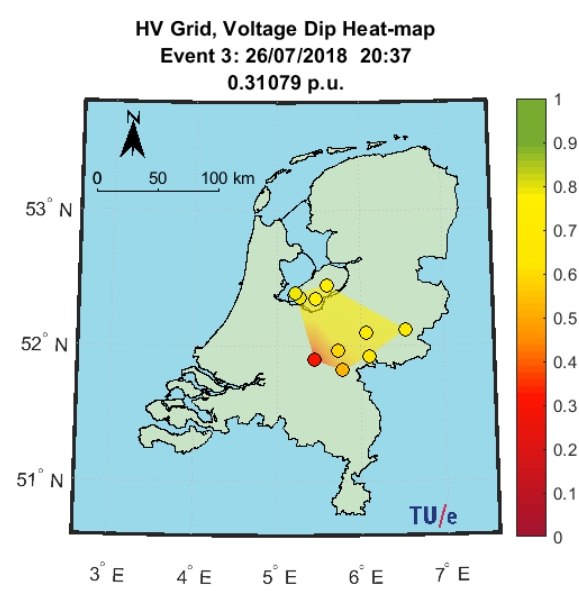

(c)

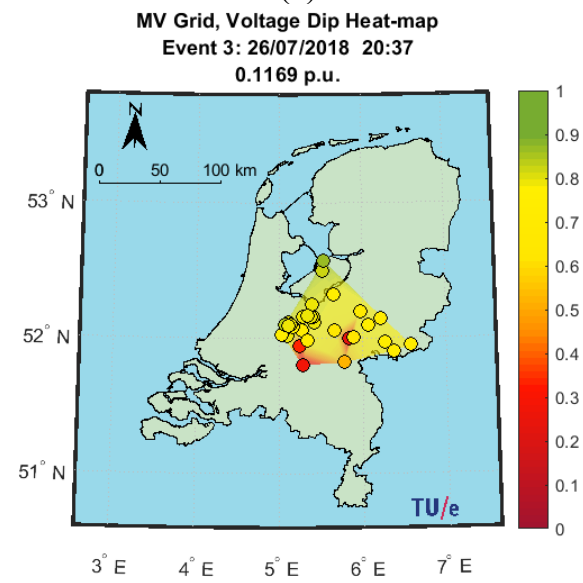

(d)

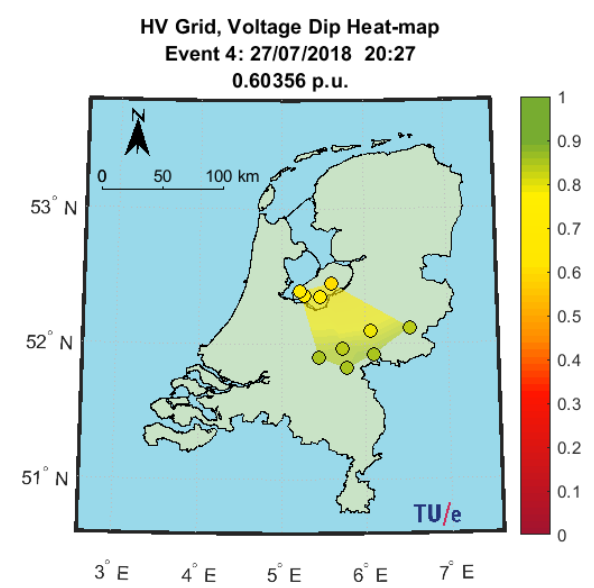

(e)

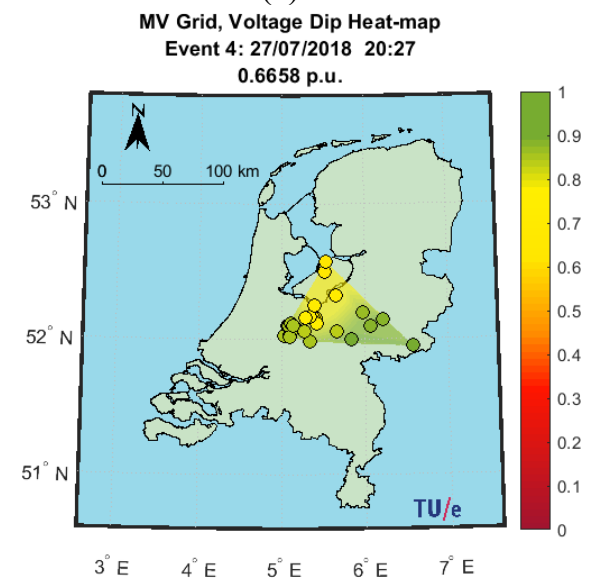

(f)

Fig. 6. The retained voltage propagation caused by Event 2, 3 and 4 from the Event List.

\section{Conclusion}

Emerging power system is highly influenced by the ET. Therefore, it is essential to investigate the effects of the ET on the operation of the future power system. The aim of this paper is to cast light on the impact of the ET on the power quality of the grid, especially, the impacts of changes in short-circuit level on the voltage dips, by proposing a step-wise approach.

The introduced step-wise approach considers various aspects for analysing the impacts of generation shift from centralized large-scale synchronous generation to RESs in the Dutch electricity grids by concentrating on the voltage dips. The possible challenges of each step are investigated and possible solution to overcome these obstacles are discussed. Moreover, the existing grid status from voltage dip perspective is investigated using PQMs data. A shortlist of candidate voltage dip events to be regenerated results of this investigation.

\section{Acknowledgement}

This work is supported by Netbeheer Nederland. The authors gratefully acknowledge the valuable comments from the ACM (Dutch Regulator) and all connectedparties. We are also grateful to Movares for supporting the analysis of the measurement data. Finally, many thanks to the TenneT colleagues for the useful technical discussions and contributions.

\section{References}

[1] S. Dennetiere, H. Saad, Y. Vernay, P. Rault, C. Martin, and B. Clerc, "Supporting Energy Transition in Transmission Systems: An Operator's Experience Using Electromagnetic Transient Simulation," IEEE Power Energy Mag., vol. 17, no. 3, pp. 4860, 2019.

[2] Netbeheer Nederland, "Code amendment proposal with regard to the standardization of voltage dips in medium and high voltage networks," 2017.

[3] M. Eliassi, P. A. B. Paulino, R. Torkzadeh, and P. Rodriguez, "Event-Based Under-Frequency Inertia Emulation Scheme for Severe Conditions," in 7th International IEEE Conference on Renewable Energy Research and Applications, ICRERA 2018, 2018.

[4] European Commission, "Network code on requirements for grid connection of generators," no. July 2009, pp. 1-68, 2016.

[5] ACM, "Netcode elektriciteit," (In Dutch), 2020. [Online] Available: https://wetten.overheid.nl/BWBR0037940/2020-0101. [Accessed: 21-Jan-2020].

[6] M. Speychal, M. H. J. Bollen, and K. Lindén, "Impact of increasing amounts of wind power on the dip frequency in transmission systems," in Nordic Wind Power Conference, 2006, pp. 22-23.

[7] M. Speychal, M. H. J. Bollen, and K. Lindén, "Impact of increasing levels of DER on the dip frequency in transmission systems," 2006 9th Int. Conf. Probabilistic Methods Appl. to Power Syst. PMAPS, pp. 1-6, 2006.

[8] S. Altschäffl and R. Witzmann, "Effect Of Reduced Rotating Inertia On Expansion Of Voltage Dips Caused By Three-Phase Faults In The German Power Transmission Network," no. June, pp. 15-18, 2015.

[9] TenneT and Gasunie, "IP2020 scenariobeschrijving," (in Dutch), 2019. 